\title{
Acerca del ciclo evolutivo del Trypanosoma (Schizotrypanum) cruzi Chagas 1909, en sus fases tisular y hematica*
}

\author{
Cecilio Romaña \\ Instituto de Medicina Regional, Resistencia, Argentina \\ (Com 5 figuras no texto)
}

No obstante los años transcurridos desde el descubrimiento de la enfermedad de Chagas y los estudios realizados sobre el Trypanosoma (Schizotrypanum) cruzi Chagas 1909, que la produce, existen aún diferencias de opinión sobre el ciclo evolutivo por el cual pasa el flagelado después de su ingreso en los organismos vertebrados.

Particularmente en los últimos años el tema ha vuelto a ser considerado en diversas publicaciones que han aportado interesantes elementos de juicio al problema.

Conviene antes de referirse a tales trabajos recapitular las opiniones vertidas anteriormente por los primeros autores que se ocuparon del asunto.

Carlos Chagas, desde el comienzo de sus estudios, se interesó por saber qué es lo que ocurría con los flagelados que, de los triatóminos transmisores penetraban en el organismo del hombre y de los mamíferos sensibles.

En su primera publicación de 1909 acertó en señalar que el tripanosoma se multiplicaba en la intimidad de los tejidos, habiendo sido conducido a esta conclusión correcta por una confusión con otro parásito que infectaba los animales de experimentación que utilizaba. Tomó por formas hísticas del $T$. (S.) cruzi formas de esquizogonia pulmonares del Pneumocystis carinii.

En el mismo trabajo refiere Chagas el modo en que los tripanosomas sanguineos pasan a la forma de multiplicación intracelular. Describió el fenómeno en los capilares del pulmón donde los tripanosomas perderían el flagelo y la membrana ondulante, se curvarían para adquirir primero una forma semilunar, para luego fundir-se en una forma esférica con núcleos y blefaroplasto; luego continúa CHaGas referiendo la multiplicación esquizogónica, que veía en sus preparados.

\footnotetext{
* Recebido para bublicação a 25 de Outubro de 1955.
} 
Es indudable que la primera parte del fenómeno, es decir el acortamiento, el doblamiento sobre si mismo y la pérdida de flagelo y membrana ondulante fueron observados sobre tripanosomas, como se aprecia en los dibujos que publicó (pág. 177 del trabajo citado); habiendo estudiado la faz esquizogónica posterior sobre Pneumocystis según se vé en los mismos diseños.

Los fundamentales estudios de GaSPar Vianna, 1911, ayudaron rápidamente a Chagas a rectificar parte de las conclusiones a que había llegado en sus primeras publicaciones, reconociendo que la multiplicación intracelular del parásito se realizaba también por un mecanismo de división binaria.

En el trabajo que Chagas publicó en 1911 acepta ya las ideas de VianNa, quien hace conocer sus investigaciones en el mismo número de las Memórias. Sin embargo, ChaGas nunca abandonó por completo su primitiva idea de la multiplicación esquizogónica, considerando que coexisten las dos formas de multiplicación intracelular del parásito: la esquizogónica y la división binaria. Además sostuvo desde sus primeras investigaciones que había dos mecanismos en la formación de jóvenes parásitos, tanto en el huésped vertebrado como en el triatómino transmisor: por gametogonia, es decir por multiplicación sexuada y por multiplicación asexuada. Impresionado por la existencia concomitante de dos formas sanguineas del flagelado facilmente deferenciables, consideró a los tripanosomas anchos formas hembras y a las finas y largas tripanosomas machos. La unión sexual se realizaría en el aparato digestivo del triatómino, siguiéndose luego el ciclo sexual en este insecto con un mecanismo semejante al que ocurre en la malaria.

También Chagas señaló la existencia de formas endoglobulares del tripanosoma, formas endoglobulares que posteriormente han sido consideradas como ejemplares libres superpuestos sobre glóbulos rojos y erróneamente interpretados en los preparados ${ }^{1}$.

GaSPAR Vianna (1911) y HaRTMANN (1911) fueron quienes por primera vez señalaron correctamente las formas intracelulares del $T$. (S.) cruzi mostrando que podían ser halladas bajo figura de leishmania y también bajo forma flagelada. El primero estudió muy bien la multiplicación de los parásitos en cortes histológicos de autopsias humanas y de animales de experimentación, observando el proceso en el interior de las células, el relleno del protoplasma con las formas de multiplicación y por último la ruptura de la membrana celular con la salida de los

1 Sin embargo conviene notar aquí una observación que hemos realizado hace poco tiempo sobre sangre de tatú (Dasypus novemcintus) muy parasitado por $T$. (S) cruzi. Siguiendo por varias horas en la platina del microscopio, gotas frescas de sangre, hemos visto la penetración de tripanosoma al interior de glóbulos rojos $\mathrm{y}$ hemos podido observar que, en tales condiciones, el flagelado se mantiene endoglobular y aún inicia un proceso de regresión hacia leishmanioide. Ahora bien, nunca hemos podido observar parásitos endoglobulares en preparaciones de sangre recién recogidas. Es posible que los hallazgos descritos por CHAGAS, lo fueron sobre sangre parasitada no fijada rapidamente y mantenida algún tiempo fuera del organismo infectado, es decir en condiciones mas o menos semejantes a los que originaron nuestra observación. 
protozoarios; haciendo notar, cuando se refiere al tejido muscular, que “... parásitos, unos todavía redondos, otros ya flagelados son lanzados en los intersticios de las fibras musculares".

VIANNA, como consecuencia de sus observaciones, dió en forma concisa y clara el esquema fundamental del ciclo evolutivo del T. (S.) cruzi en los tejidos vertebrados, al decir: "De lo que observamos podemos afirmar que el tripanosoma penetra en la célula, multiplícase por división binaria, rompe la membrana y sale..."

Con posterioridad fué Brumpt (1912) quien estudió el problema. Este autor consideró las formas anchas, tripanosomas adultos, rechazando en tal forma la teoría sexual sostenida por CHAGAS. En su trabajo dió el esquema del ciclo evolutivo del $T$. (S.) cruzi que aún permanece inalterado en su manual de parasitología.

El trabajo de Mayer y Rocha Lima (1914) constituye la siguiente publicación fundamental. En cortes histológicos de animales infectados estudiaron el ciclo vertebrado del parásito reconociendo los aspectos ya señalados por VianNa y HaRTMANN, es decir las formas leishmanias y las formas flageladas intracelulares. Sin embargo notaron que las formas leishmanias podían presentarse ya redondeadas, ya alargadas en forma de huso.

Describen la transformación de las formas leishmanias en tripanosomas a partir de las formas redondas por desenrollamiento ("aufrollung") en los siguientes términos: "el flagelo se curva y rodea al parásito redondeado, casi como una membrana de quiste y después el parásito se desarolla para formar el parásito definitivo". La descripción está bien ilustrada por figuras que muestran las fases sucesivas. En los parásitos fusiformes, que encuentran ser poco frecuentes, señalaran que primero aparecen formas de critidias y luego tripanosomas, probablemente por migración del blefaroplasto, como en Trypanosoma lewisi. Si bien señalan que la mayoría de los focos de multiplicación parasitaria contienen sólo formas leishmanias o sólo formas flageladas, notan que existen conglomerados donde pueden observar-se ambos tipos simultáneamente como ya los había señalado VIANNA.

En cuanto a WeNYoN (1926) repite los conocimientos expuestos por Chagas, Vianna, Brumpt, etc. Si bien no da en el texto de su tratado de protozoología una especial referencia a los dos descritos por MAYER y Rocha Lima, en la pág. 491 ofrece una figura donde el desarrollo desde la forma leishmania hasta tripanosoma se sigue según dos sistemas de crecimiento: uno en que los parásitos conservan una forma redondeada hasta llegar a tripanosomas y otro con progresivo alargamiento en forma de huso; interpretación obtenida personalmente por WENYON sobre una preparación de frotis de corazón de laucha enviada por el Dr. Tejera de Venezuela.

Ni la tesis de Niño (1929) ni la de Dias (1934) aportan nueva información al conocimiento del ciclo evolutivo del Trypanosoma (S.) cruzi en el huesped vertebrado; limítanse estos autores a referir las informaciones ya expuestas por Chagas, Vianna, MaYer y Rocha Lima, BRUMPT, etc. 
En 1935 KofoId, Wood y Mc NeIL publican el primer intento de seguir el ciclo del $T$. (S.) cruzi en cultivo de tejidos de corazón de embrión de rata y laucha blanca. Estos autores observaron la aparición de los primeros tripanosomas al quinto día de la inoculación bajo la forma de flagelados agrupados en las vecindades de la masa central del preparado. No pudieron practicar observación directa sobre el ciclo de transformación de los parásitos intracelulares.

En 1942 ROMã̃a y MEYER intentan nuevamente cultivo de $T$. (S.) cruzi en tejidos de embrión de gallina, usando la técnica de gota pendiente. Bajo observación directa pudieron observar la penetración de tripanosomas metacílicos de cultivo en agar-sangre en el interior de fibroblasto y miocitos del cultivo de tejido.

Observaron que los flagelados ya en el protoplasma celular reducen sus movimientos que quedan localizados en la membrana ondulante y el flagelo; 20 horas después hay desaparición de la membrana ondulante â gran acortamiento del flagelo libre. Al mismo tiempo el cuerpo se acorta, volviéndose globuloso. Algunas horas más tarde el parásito se presenta bajo forma leishmanioide. Después de este momento no pudieron seguir por observación directa los tripanosomas por desaparecer estos en el protoplasma celular, con igual índice de refringencia. En preparaciones coloreadas es posible observar que la transformación se acompaña de una migración del blefaroplasto hacia la extremidad anterior del parásito así como la transformación del corpúsculo de redondeado en un bastoncito.

A los tres días se pueden descubrir los parásitos transformados nuevamente en flagelados que denuncian su presencia por el movimiento de sus cuerpos, membranas ondulantes y flagelos.

Los autores refieren otro mecanismo de transformación hacia leishmanioide después que los flagelados han penetrado en las células: vieron que critidias y tripanosomas se enrollan sobre sí mismos sufriendo la evolución hacia leishmanioide en esta posición.

La división binaria fué observada en leishmanioides y también en formas ya flageladas (critidias). Nunca fueron observadas figuras en división múltiple ni tripanosoma en división binaria.

Los autores refieron luego que: "Los movimientos de los flagelados intracelulares son cada vez mas activos hasta que, la célula completamente llena de parásitos, sólo muestra tripanosomas en movimiento. En las láminas coloreadas obsérvase la transformación de las formas critidia en tripanosomas con migración del blefaroplasto hacia la parte posterior del cuerpo, y el desarrollo de la membrana ondulante.

"Al $4 .^{\circ}$ día ya pueden aparecer algunos tripanosomas libres pero en general es en el $5 .^{\circ}$ día que se observan los primeros. Pasan al medio externo gracias a la brusca ruptura de la célula que los contiene. En este momento se pueden observar, junto a formas que alcanzaron el desenvolvimiento completo de tripanosomas, otras que todavía están en forma de critidia y aún de leishmanias".

Refieren los autores que los tripanosomas adultos que salen de las células son de dos tipos semejantes a los que se encuentran en la co- 
rriente sanguínea de los vertebrados: algunos son finos y largos y otros anchos. Desde el principio observáronse ambas formas en las culturas libres e intracelulares. Los tripanosomas finos muestran un tipo de movimiento de progresión ondulatoria en linea recta semejante a los tripanosomas metacíclicos, mientras las formas anchas tienen movimento sobre sí mismo sin gran desplazamiento del lugar donde se mueven.

También observaron que en ciertas células parasitadas existen formas redondas de leishmanias que rápidamente se transforman en tripanosomas adultos por desarrollo del flagelo sin migración del blefaroplasto, seguido de desenrollamiento del protozoario sin que se pueda observar la fase intermedia de critidia. Aspectos de esta transformación van a ser comentados nuevamente más adelante, en el curso de este trabajo.

ElKELES en 1940, 1942, 1943, 1951, ha sostenido diversas opiniones sobre el ciclo evolutivo del $T$. (S.) cruzi. Sería difícil seguir en detalle sus trabajos que están fundados principalmente en sus observaciones sobre cultivos "in-vitro" de $T$. (S.) cruzi lo cual escapa al objeto de nuestro presente estudio. Principalmente ElkELes reactualizó las teorías de multiplicación sexual y esquizogónica sostenida por C. Chagas.

A nuestro critério, la observación mas interesante de su trabajo de 1942 reside en llamar la atención sobre el pasaje directo de formas leishmanias a tripanosomas sin faz intermediaria de critidia como ya lo habían señalado MAYER y Rocha LIMA (1914) en cortes histológicos y Romaña y MeYer (1942) en cultivos de tejidos.

ELKELES publica en 1951 un artículo en que reactualiza su argumentación sobre diversos aspectos del ciclo evolutivo del $T$. (S.) cruzi. En este trabajo sostiene que no puede aceptarse la existencia de una faz critidio-morfa durante el desarrollo del $T$. (S.) cruzi en vertebrados y que los tripanosomas se forman a partir de las leishmanias redondas sin existencia de una faz critidia, opinión que generaliza a cualquier medio donde se desarrollan formas tripanosomas.

En 1945 Pizzi estudia en perritos jóvenes intensamente parasitados formas evolutivas de $T$. (S.) cruzi en los tejidos. Halla muchos nidos de flagelados formados por critidias o tripanosomas aisladamente pero en ciertos casos halla juntos, en el mismo conglomerado, ambos estados del parásito.

En 1951, S. F. Wood estudió una raza de $T$. (S.) cruzi procedente de Arizona que producía intensas infecciones en ratones (Mus musculus).

Las lauchas eran sacrificadas y con los órganos se efectuaban preparaciones por "impresión" que secas y coloreadas le permitieron concebir el ordenamiento en que se produce la evolución del $T$. (S.) cruzi en el huésped vertebrado.

$\mathrm{El}$ autor quedó impresionado por la ausencia de formas critidias en las figuras que observó, en desacuerdo con los conocimientos considerados clásicos (BRUMPt, MAYer y Rocha LIMA, Dias, etc.) quienes siempre señalan la existencia de esta faz flagelada. 
Wood llegó a la conclusión de que la forma de leishmania intracelular evoluciona hacia tripanosoma siguiendo dos mecanismos: en el primero, al cual llama "directo", se produce un alargamiento de los leishmanioides fusiformes, con crecimiento del flagelo según el eje longitudinal y desarrollo paralelo de la membrana ondulante. En tal condición el kinetoplasto, colocado desde un principio en la extremidad posterior del parasito, no sufriría la migración señalada por BRUMPT, desde la parte anterior hacia la parte posterior del núcleo.

Por este simple proceso de alargamiento el leishmanioide se transformaría en tripanosoma sin pasar por una faz intermedia de critidia.

El segundo mecanismo al cual Wood llama "indirecto", tiene origen en los leishmanioides redondeados ya señalados por MAYER y RocHA LiMA. La evolución se realizaría por crecimiento del flagelo alrededor del protoplasma, aparición de una muesca en la parte interior del protozoario y desarrollo de la membrana ondulante y del flagelo libre, con la cual se completaría la formación del tripanosoma. Este mecanismo corresponde a lo que MAYBR y Rocha LIMA han llamado "aufrollung" y que dichos autores vieron en cortes histológicos.

Wood ilustra con figuras esquemáticas ambos mecanismos evolutivos cuya descripción hemos dado en forma muy sintética, pués el autor entra en numerosos detalles acerca de las transformaciones que sufren las diversas organelas del protozoario.

Wood encuentra un mecanismo de desarrollo "directo" en las figuras 10 a 15 que presenta WenYon (1926) en la página 491 de su Protozoology, figuras que no concuerdan exactamente con la interpretación dada por el primero, pues WeNYoN dibuja una migración del kineplasto hacia la extremidad posterior de acuerdo a la teoría clásica, lo cual envuelve un pasaje por forma critidia, que, como hemos dicho, Woon no acepta en su teoría.

Em 1953 Wood amplió sus estudios de 1951 en un trabajo que pretende, ayudado por métodos hematológicos, seguir la evolución completa del T. (S.) cruzi en el huésped vertebrado, es decir la transformación de tripanosoma en leishmanioide, y da evolución de leishmanioide nuevamente en tripanosomas. A la primera faz, llama desarrollo "regresivo" y a la segunda "progresiva" 2.

El principal elemento de juicio que considera Wood para determinar si un parásito se encuentra en período "progresivo" o "regresivo", reside en la afinidad tintorea del protoplasma; una tendencia basófila significa una evolución "regresiva" y una acentuada afinidad acidofila acompaña al período "progresivo".

2 Con objeto de no crear nuevos términos usaremos en nuestra exposición las palabras aqui empleadas por Wood para referirse a las faces del ciclo del $T$. (S.) cruzi aún cuando compartimos la opinión de GaLliard (1952) quien dice: “... la régression ou rétrogréssion, termes impropres et péjoratifs, puisque après tout, c'est la forme leishmania qui, se multipliant, est la forme noble". 
Estas modificaciones tintoreas se acompañarían, cuando la evolución es "regresiva", de: aumento de gránulos de volutina, y vacuolización del protoplasma, arredondeamento del núcleo y aspecto en creciente del cuerpo del parásito.

De acuerdo con estas ideas Wood señala la existencia de tres tipos de tripanosomas en la sangre periférica: "progresivos cortos y finos", "progresivos largos y finos" y "regresivos anchos". Los tripanosomas finos serían más abundantes en la sangre al principio de las infecciones. Al prolongarse la infección disminuyen en número por transformación en tripanosomas anchos más viejos.

El autor explica la formación de las formas por las presiones que sufren los parásitos en células alargadas como las musculares $p$. ej. lo cual conduce a un desarrollo longitudinal del parásito (método directo de Wood). Si las presiones se realizan en un área oval o redonda el flagelado se desarrolla bajo la forma circular (método indirecto de Wood) .

En 1952 Galliard ha publicado un interesante trabajo en que vuelve sobre antiguas observaciones efetuadas sobre un rico material obtenido en lauchas infectadas con $T$. $(S$.$) cruzi.$

Esta raza de tripanosomas tenía la particular característica de multiplicarse intensamente en los músculos de la cintura abdominal y de encontrarse gran quantidad de ejemplares adultos en la cavidad peritoneal y en el líquido edematoso que embebía las mallas del tejido conjuntivo vecino al peritoneo y aún subcutáneo, mientras en la sangre eran escasos o no se encontraban.

Galliard sostiene que el estudio del ciclo evolutivo parasitario en frotis o impresiones de tejidos de acuerdo a como lo realiza Woon, falsea la correcta interpretación del fenómeno, pues no es posible establecer filiaciones ni reducir sucesión de formas existentes en los focos de multiplicación intratisular y en el exudado extracelular, por el sólo hecho de ser las figuras vecinas en los frotis de tejidos. Sostiene que unicamente puede sacarse conclusiones del estudio de los cortes histológicos. Critica en la pag. 82 a quienes ven una doble forma de evolución del $T$. (S.) cruzi en el huésped vertebrado basándose en el estudio de material obtenido por frotis de órganos diciendo "C'est grâce à cette méthode qu'on a pu décrise deux cicles évolutifs chez le vertébré, ce qui est difficile à concevoir et ne correspond pas à une nécessité biologique, à moins que l'on n'admette l'existence d'un processus sexué, ce que nous garderions bien de faire dans l'état actuel de nos connaissances".

En definitiva GaIllard cree que las grandes diferencias señaladas por diversos autores en la morfología y la evolución del flagelado en el huésped vertebrado serían en gran parte meros artificios de las preparaciones, terminando que: "Par ailleurs, il parait évident qu'une même forme peut être grèle ou large, resistante ou fragile, suivant la technique que l'on emploie".

Después de haber pasado en revista los principales trabajos que han sido dedicados al estudio del ciclo evolutivo del $T$. (S.) cruzi en 
vertebrados podemos concluir que los autores mas recientes (Romaña y MeYer, Wood y ElKelesq aceptan, en general, las ideas de Meyer à Rocha Lima en lo que se refiere a la transformación intracelular de leishmaniodes en tripanosomas, fenómeno que podría ocurrir por uno de los dos mecanismos siguientes: 1) por simple alargamiento del leishmaniode bajo aspecto fusiforme $\hat{a}$ 2) por desenrollamiento de formas redondas que crecen curvadas sobre sí mismas en los focos de multiplicación intraprotoplasmáticos.

Cuando se refieren a las formas de transición redondas, estos autores están de acuerdo en que el fenómeno se realiza por un desarrollo del fragelo y membrana ondulante alrededor de la masa protoplasmática a la cual circunda sin que exista migración del kinetoplasto. Como consecuencia, haâ un pasaje directo de leishmanioide a tripanosoma sin una faz critidia intercalada.

Cuando la evolución se realiza bajo forma fusiforme, Wood, sostiene que tampoco existe migración de kinetoplasto y como consecuencia falta igualmente la faz critidia. Entretanto, RoMaña y MEYER estudiando la evolución fusiforme, basados en sus observaciones de cultivos de tejidos, sostienen la existencia de una faz critica intermedia entre leishmanioides y tripanosomas.

Es nuestra intención en el presente artículo reconsiderar puntos de vista y tratar de ordenarlos de acuerdo a la información existente y a nuestra experiencia personal .

\section{MATERIAL DE OBSERVACIÓN}

Como elemento de observación hemos dispuesto del numeroso material histológico existente en la colección del Instituto de Medicina Regional; de cortes de tejidos de un mono Callithrix sp., infectado por vía ocular con deyecciones de Rhodnius prolixus (Instituto Pasteur, Laboratorio del Prof. Roubeaud, París 1939); de preparaciones de cultivos de tejidos infectados con $T$. (S.) cruzi y realizados en el Instituto de Biofísica de la Facultad de Medicina de Río de Janeiro (en colaboración con la Srta. H. Meyer) y en el Instituto de Medicina Regional con la ayuda de la Srta. L. Scheffls y por último, con preparaciones de impresiones de órganos de oculto (Ctenomys tucumanus) infectados por inoculación de deyecciones de Triatoma infestans locales.

La historia del mono Callithrix ha sido referida en un trabajo anterior (1939), la técnica de los cultivos de tejidos infectados con T. (S.) cruzi está consignada en el trabajo realizado en colaboración con la Sta. MeYer (1941); la misma forma de preparación de cultivos de tejidos se utilizó en el Instituto de Medicina Regional; en cuanto a las infecciones de Ctenomys tucumanus, Mazza ha señalado la gran sensibilidad de estos roedores para las infecciones por el $T(S$.$) cruzi así$ como el abundante desarrollo de parásitos tisulares y hemáticos que en ellos puede observarse; otro tanto ocurrió con los animales por nosotros inoculados que nos suministraran frotis de órganos para la observación de los parásitos. 
Consideramos que las observaciones realizadas en cultivos de tejidos son fundamentales para seguir el desarrollo intratisular del $T$. (S.) cruzi pues se reproducen aproximadamente las mismas condiciones existentes en el vertebrado, y, gran parte de la evolución del flagelado puede seguirse directamente bajo el microscopio, en fresco.

Consideramos que las impresiones de órganos y los cortes histológicos no son sino métodos auxiliares que ayudan al método anterior.

\section{PUNTOS DE VISTA DEL AUTOR}

De acuerdo a nuestras observaciones en cultivos de tejidos los tripanosomas metacíciclicos de cultivo o los tripanosomas sanguíneos infectantes penetran activamente en el protoplasma de las células y allí se van inmovilizando lentamente en un proceso regresivo que aparentemente se realiza de acuerdo a dos mecanismos diversos: 1) regresión fusiforme ${ }^{3}$ : el tripanosoma ya en el interior del protoplasma celular, conserva su forma alargada, pierde su movimiento de translación pero continúa agitado por movimientos ondulatorios especialmente a nivel de la membrana ondulante y del flagelo libre. El cuerpo se ensancha y acorta y la parte libre del flagelo se reduce rápidamente de longitud. A las 24 horas tiene ya una configuración de leishmania que no puede diferenciarse, a fresco, del protoplasma celular que la rodea.

En las preparaciones coloreadas se ve que se ha producido una migración del kinetoplasto hacia la parte anterior del protozoario tomando una configuración de barra ligeramente curvada y que el flagelo ha quedado reducido a un pequeño tamaño, siendo siempre visible en las buenas preparaciones. 2) regresión orbicular: en nuestro trabajo en colaboración con la Sta. MEYer (1942) la hemos descrito en los siguientes términos: "En numerosas culturas de tejidos infectados con T. (S.) cruzi procedentes de culturas en agar-sangre o de otra cultura de tejido, vimos que critidias o tripanosomas después de penetrar en las células o en el medio externo, se enrollan sobre si adquiriendo en las preparaciones coloreadas, el aspecto de leishmanias. En ciertos casos este fenómeno ocurre 15 minutos después de la contaminación. Hemos

3 Hemos llamado fusiforme y orbicular las dos formas de evolución regresiva que se observan en el $T$. ( $S$.) cruzi. Estas designaciones definen morfologicamente el aspecto general del fenómeno sin entrar a considerar los detalles de su desarrollo interno. Estas voces las usamos igualmente al describir las fases progresivas en las cuales WooD emplea las palabras "directa" e "indirecta". Creemos que "fusiforme" y "orbicular" son preferibles pues no se prestan a confusión al no pretender explicar el mecanismo del fenómeno. P. ej., para nosotros, en oposición a WooD, la transformación de leishmanioide en tripanosoma por "desarrollamiento" seria el verdadero mecanismo "diracto" pues no existe una faz intermedia de critidia.

El diccionario de la Real Academia Española define "fusiforme" como "de figura de huso" y "orbicular" como "redondo o circular". 

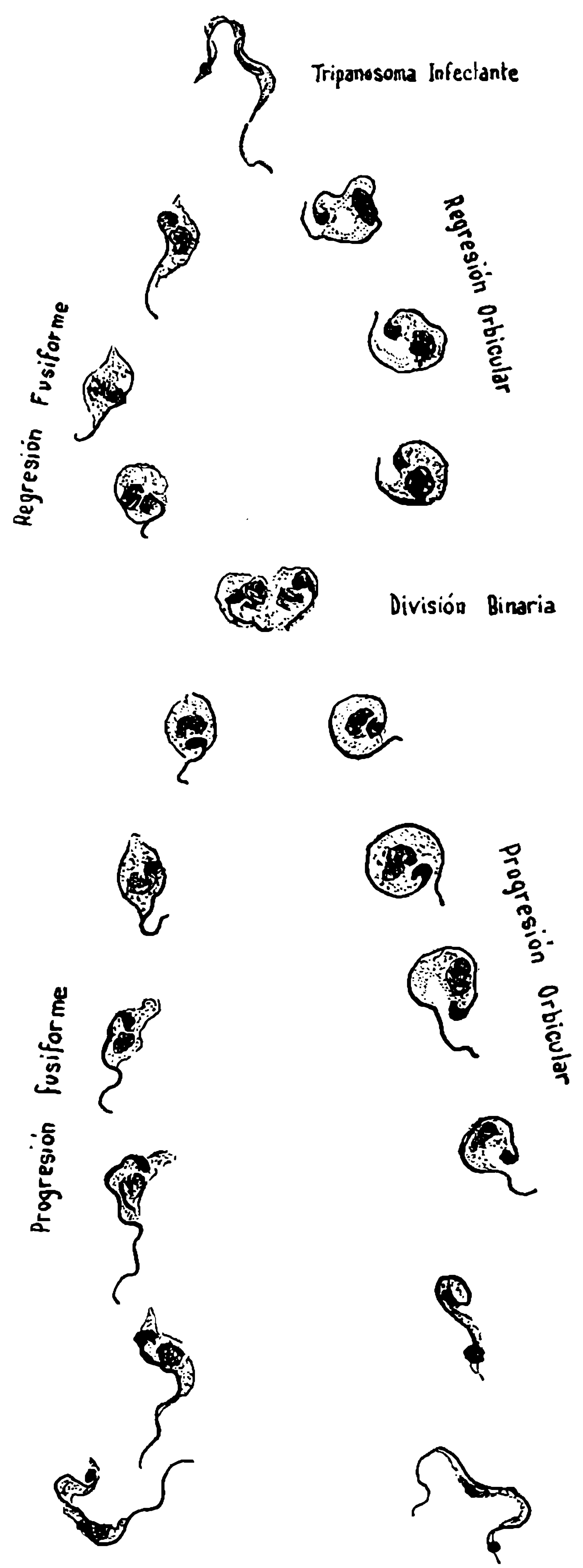

Fig. 1 - Ciclo evolutivo T. (S.) cruzi en los tejidos de vertebrados. Interpretación del Dr. Romaña. 
observado también el hecho con tripanosomas originarios de la misma cultura, los que también se enrollan dentro o fuera de las células. Parecería que las formas intracelulares evolucionaran hacia leishmanioides en esta posición enrollada, siguiendo mas tarde el ciclo evolutivo ya descrito. Las formas extracelulares parecen degenerar y mueren".

Una vez que los tripanosomas infectantes se han transformado en leishmanioides en el interior de las células se inicia la multiplicación del parásito por división binaria como lo señalara por primera vez VIANNA.

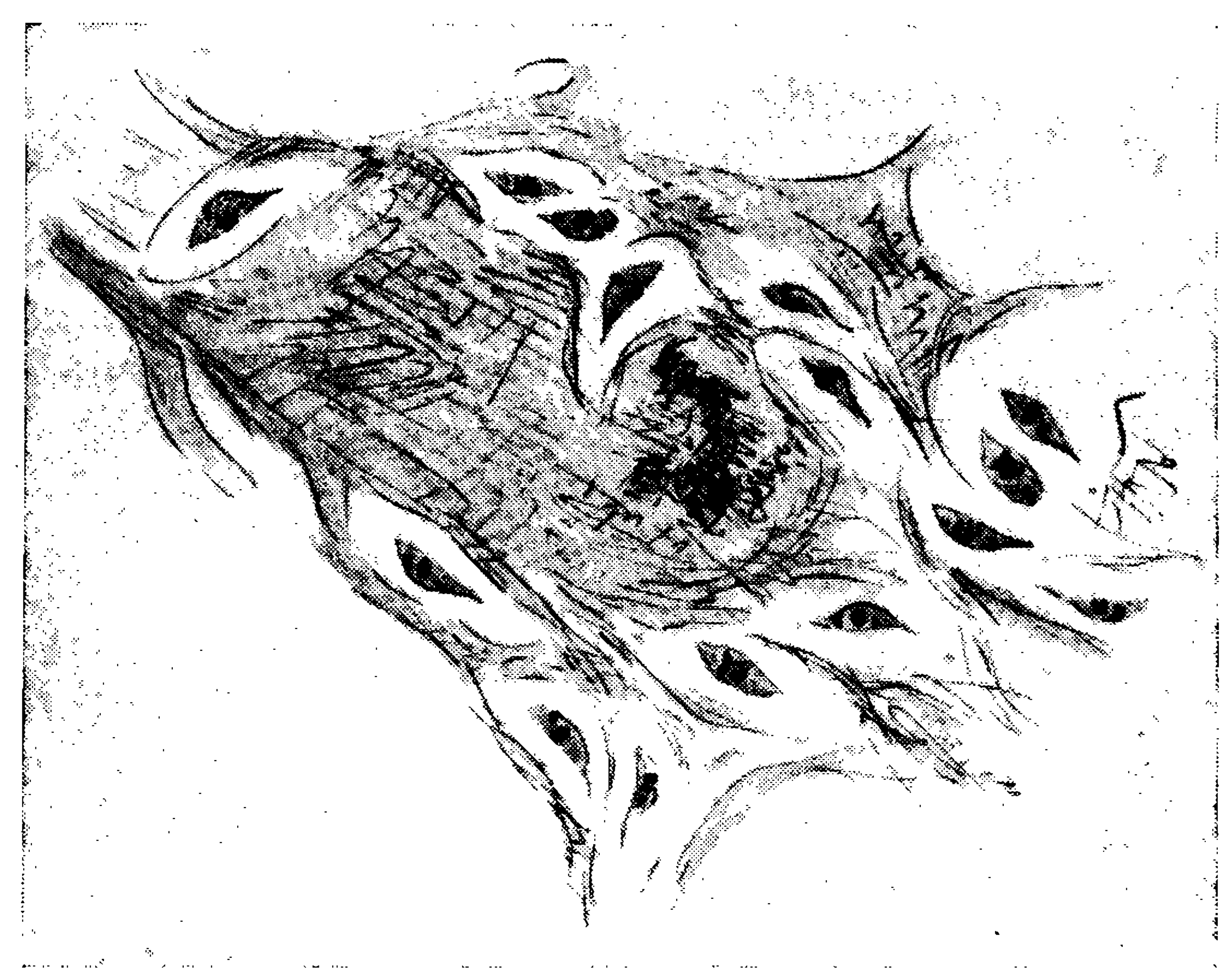

Fig. 2 - Formas fusiformes

En los cultivos de tejidos se nota que este fenómeno se realiza rapidamente, llenándose frecuentemente en sólo 48 horas el protoplasma celular.

H. MeYeR observó que el completo rellenamiento del protoplasma marca el comienzo de la acelerada evolución "progresiva" de los leishmanioides hacia tripanosomas.

Como los describen MaYeR y Rocha Lima y lo han confirmado RoMAÑA Y MEYER y también WooD, esta evolución se puede realizar por dos mecanismos simultáneos: 1) la "progresión" por alargamiento y 2) "progressión" por desenrollamiento, fenómeno a los cuales por analogía con lo que ocurre en la faz regresiva llamaremos progresión fusiforme y progresión orbicular.

Progresión fusiforme: Una vez lleno el protoplasma celular con las formas leishmanioides, estas comienzan a alargarse en el sentido del 
eje longitudinal con crecimiento del flagelo y desarrollo de la membrana ondulante. Es en este momento que el protozoario adquiere una configuración semejante a critidia con el kineplasto en situación antenuclar, configuración que sólo dura poco tiempo pués la migración del kinetoplasto hacia la parte posterior del núcleo cambia el aspecto del parásito que se aproxima a la forma tripanosoma.

Wood señala que la evolución progresiva va acompañada de un cambio en la afinidad tintorea de los parásitos cuyo protoplasma se hace acidófilo.

Progresión orbicular: En forma simultánea con la "progresión fusiforme" se realiza, en ciertos elementos leshmanioides el desarrollo bajo forma "orbicular". En este caso los leishmanioides redondeados desarrollan el flagelo que circunda casi completamente el protoplasma del parásito. El flagelo crece luego fuera del cuerpo arrastando una porción de protoplasma que forma la membrana ondulante. Estando el parásito siempre enrollado se forma una muesca en su porción interna, muesca que progresando termina por dar forma alargada al cuerpo del tripanosoma, que, sin embargo, siempre se mantiene en la misma posición.

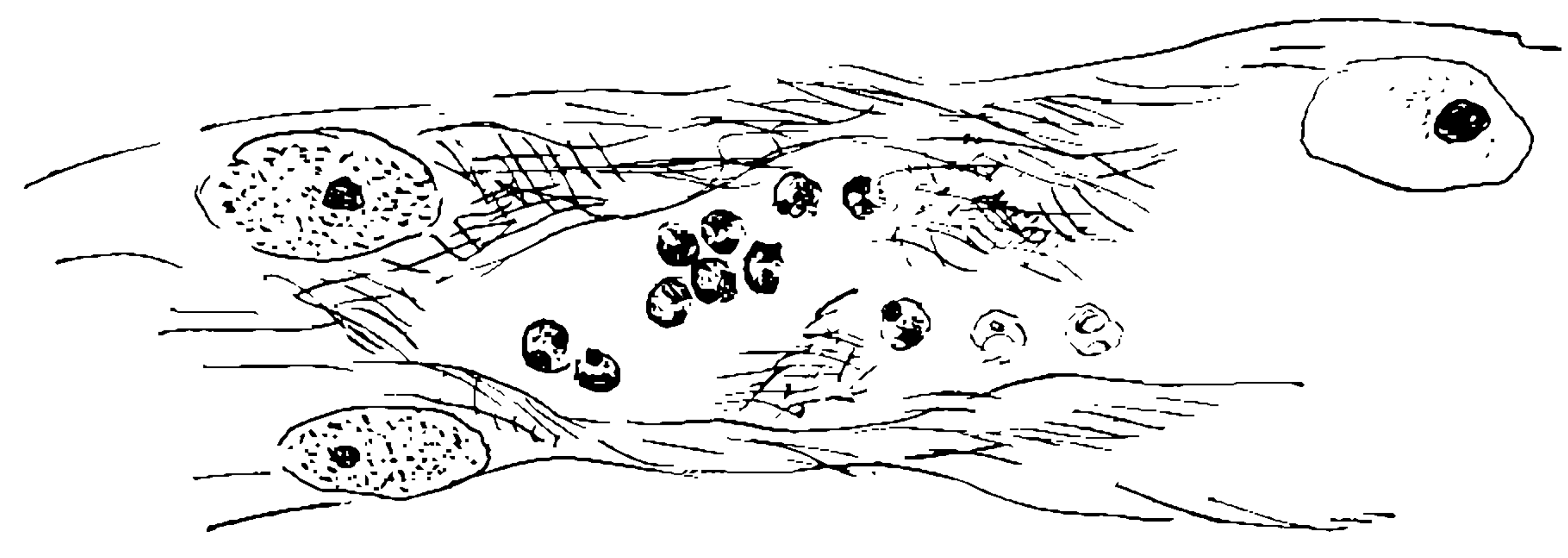

Fig. 3 - Miocardio. Formas orbiculares.

En todo el proceso el kinetoplasto no cambia de situación, permaneciendo en la parte del parásito que será más tarde, la extremidad posterior.

Dicha organela tampoco cambia mucho de forma pués en los leishmanioides redondeados el kinetoplasto no tiene la configuración en bastoncito que se halla en los fusiformes, sinó la forma en poroto o la redondeada que se encuentra en los tripanosomas que han llegado a su formación completa (WooD) .

Las diferentes faces del ciclo evolutivo anteriormente descrito han sido esquematizadas en el dibujo de la fig. N. 1, y se cumplen como es conocido (Dias, Kofoid, Wood y Mac NeIL, Romaña y MeYer, etc.) en un período de 4 a 6 días.

En las células parasitadas, por lo general se ven agrupadas sólo formas "fusiformes" (fig. 32) o sólo formas "orbiculares" (fig. 3, como si la multiplicación se hiciera separadamente, aún en la misma cé- 
lula. A veces sin embargo están mezcladas ambas formas en disposición irregular (fig. 4).

En general se observa que la mayoría de los individuos de una camada de parásitos desarrollada en el protoplasma de una célula, se encuentran aproximadamente en el mismo período evolutivo, sin embargo cuando la camada llega a la forma tripanosoma es frecuente ver que, un cierto número de indivíduos están atrasados en su evolución permaneciendo en estado de critidia y aún de leishmanioide.
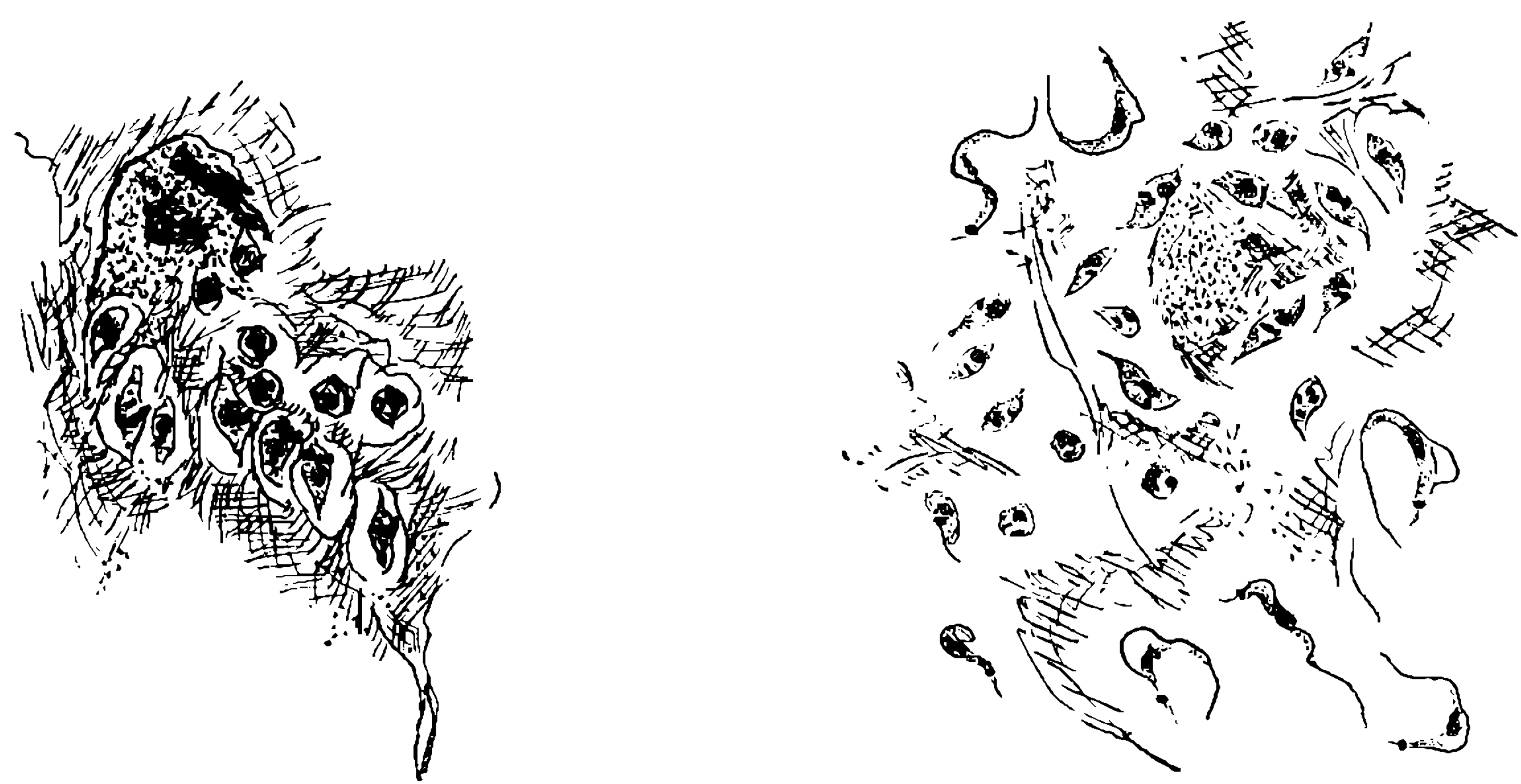

Fig. 4 (a esquerda) - Bazo; fig. 5 - Parte de los elementos parasitarios originarios de um fibroblasto recién rompido.

Ya Vianna observó que no todos los individuos de una camada se encuentran en el mismo período evolutivo, aún cuando la mayor parte lo esté. Decía Vianna: "Llegado a cierto punto de plenitud, esto es, cuando el sarcolema no puede contener más los esquizotripanos desarrollados en su interior se produce ruptura en algún punto del elemento anatómico, y, parásitos, algunos todavía redondos, otros ya flagelados, son lanzados en los interstícios de las fibras musculares".

En la fig. 5, es posible ver parte de los elementos parasitarios que han tenido origen en un fibroblasto de cultivo de tejidos que acaba de romperse. Se observan restos del núcleo, junto a detritus protoplasmáticos, además "leishmanioides fusiformes" y "orbiculares" en distinto estado evolutivo y algunos tripanosomas ya completamente desarrollados (cultivo de tejido de embrión de pollo; coloración con hematoxilina férrica) .

No obstante nuestro particular interés de poder establecer en los cultivos de tejidos si los dos tipos de progresión observados están relacionados con las dos clases de tripanosomas hemáticos, es decir los finos y los anchos, hasta ahora no hemos podido encontrar la correcta filiación de estos tripanosomas.

Sin embargo nos parece que las formas anchas son originarias en la "progresión fusiforme" y las finas en la "progresión orbicular". 


\section{RESUMEN}

El autor pasa en revista los trabajos publicados sobre el ciclo evolutivo del Trypanosoma (S.) cruzi en el huésped vertebrado, desde el descubrimiento de la enfermedad hasta nuestros días.

Luego analiza las ideas de los autores modernos, fundadas en gran parte en las observaciones que ya en 1914 realizaron MAYER y ROCHA Lima de las cuales participan actualmente Romaña y MEYeR, ELKeLES y WooD.

Finalmente expressa que a partir de los tripanosomas infectantes los parásitos que penetran en el protoplasma celular pueden seguir dos mecanismos en su evolución hacia cuerpos leishmanioides:

1.0) Por "regresión fusiforme" y 2.0) por "regresión orbicular"; llegados a la forma leishmanioide los parásitos se multiplican por división binaria, una vez lleno el protoplasma celular, siguen un proceso inverso de transformación hacia tripanosoma que puede seguir igualmente dos mecanismos diversos: 1.) "progresión fusiforme" y $2 .^{\circ}$ ) "progresión orbicular". Estos diversos mecanismos de transformación están esquematizados en la fig. N. ${ }^{\circ} 1$ del trabajo.

\section{SUMMARY}

The author reviews the published works about the evolutive cycle of the Trypanosoma cruai in the vertebrate host, from the discovery of the disease to our days.

Then, he analyzes the ideas of the modern authors who based themselves on the observations made formerly, in 1914, by MAYER \& ROCHA Lima, ideas that RoMAÑA and MEYer, Elkeles and Wood agree at the present time.

Last, he states that, from the infective trypanosomas, the parasites which enter the cellular protoplasma may follow two systems to perform their evolution up to leishmanioid bodies: $1 .^{\circ}$ ) by fusiform regression, $2^{\circ}$ ) by an orbicular regression. Once the parasites reach the leishmanioid forms, they multiply by binary division. When the celular protoplasm is filled up with the parasites, these follow an inverted transformation up to trypanosoma state, following also two systems; similar to the repression $10^{\circ}$ ) a fusiform progression, $2 .^{\circ}$ ) an orbicular progression.

The mechanism of the different transformations is sketched in figure $N .^{\circ} 1$ in the work. 


\section{BIBLIOGRAFIA}

BrUmpt, E. - 1912. Le Trypanosome cruzi évolue chez Conorhinus megistus, Cimex boueti et Ornithodorus moubata - Cycle évolutif de ce parasite. Bull. de la Société de Path. exotique, pág. 360.

Chagas, C. - 1909. Nova tripanosomiase humana. Memorias do Instituto Oswaldo Cruz 1 (3), 159-218.

Chagas, C. - 1911. Nova entidade mórbida do homem Resumo general de estudos etiológicos e clínicos, Memórias do Instituto Oswaldo Cruz, 3 (2), 219-275:

Dias, E. - 1934. Estudos sôbre o Schizotrypanum cruzi. Memórias do Instituto Oswaldo Cruz, 28 (1), 1-110.

ElKeles, C. - 1942. Investigaciones sobre el ciclo evolutivo del tripanosoma cruzi. La Semaña Médica. 49 (27), 1-16.

Elkeles, G. - 1943. Enfermedad de Chagas. Consideraciones críticas acerca de los conceptos actuales sobre el mecanismo de su transmisión al hombre y sobre el ciclo evolutivo de Trypanosoma cruzi. La Semana Médica. 50 (29), 178-186.

Elkeles, G. - 1951. On the life of Trypanosomes cruzi. The Journal of Parasitology. 37 (4), 379-386.

GalliaRd, H. - 1952. Recherches sur le cycle évolutif de Trypanosoma cruzi Chagas. À propos de l'infestation péritonéale exclusive chez la souris. $27(1-2-3), 63-85$.

Hartmann, M. - 1910. Notiz über eine weitere Art der Schizogonie bei Schizotrypanum cruzi (Chagas). Arch. Protist. 20: 361-363.

Koford, C. A., Wood, T. O. y Mc. NeIL, E. - 1935. The cycle of Trypanosoma cruzi in tissue culture of embryonic hearthmuscle. Zoology. 41 (3) 23-24.

MaYer, M. y Rocha Lima, H. - 1914. Zum Verhalten von Schizotrypanum cruzi in warm Blütern und Arthropoden. Arch. Schiffs u. Tropen Hyg. 18: $101-136$.

Niño, F. L. - 1929. Contribución de la enfermedad de Chagas. Tesis. Imprenta de la Univ. de B. Aires 1-129.

PIzzI, T. - 1945. Algunos aspectos de la enfermedad de Chagas experimental. Biológica. III. 53-68.

Romañ, C. y Meyer, H. - 1942. Estudo do ciclo evolutivo do Schizotrypanum cruzi em cultura de tecidos de embriāo de galinha. Memórias do Instituto Oswaldo Cruz. 37 (1). 19-27.

Vianna, G. - 1911. Contribuição para o estudo da anatomia patológica da molestia de Carlos Chagas. 3 (2) . 276-294.

Wenyon, C. M. - 1926. Protozoology. London, Bailiere, Tyndall and Cox. 1-1563.

Wood, S. F. - 1951. Development of Arizona Trypanosoma cruzi in mouse muscle. The Amer. Journ. of Trop. Med. and Hygiene. 31 (1), 1-8.

Wood, S. F. - 1953. Hemologic differentiation of the intramuscular developmental forms of Trypanosoma cruzi Chagas. The Amer. Journ. of Trop. Med. Hygiene. 32 (6), 1015-1 034. 